\title{
Epidemiological and serological profiles of TORCH infection in pregnancy
}

\author{
Pradhan SV ${ }^{1}$ \\ ${ }^{I}$ Advance Pathology Laboratory, Jhapa, Nepal
}

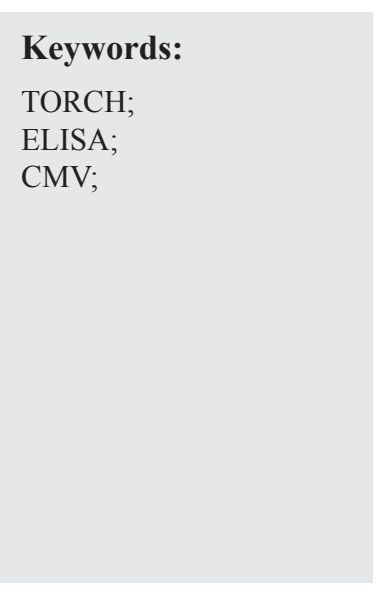

\begin{abstract}
Background: Maternal infections caused by TORCH [Toxoplasma gondii, Rubella virus, Cytomegalovirus (CMV), Herpes simplex virus (HSV)] are the major causes of bad obstetric history. The aim of this study was to evaluate the prevalence of TORCH infections in pregnancy.

Materials and Methods: The study included 109 pregnant women with unfavorable previous pregnancy. Serological evaluation for TORCH infections was carried out by IgM Enzyme Linked Immunosorbant Assay method. Data was analyzed using SPSS 17.0 whenever necessary.

Result: The IgM/ IgG sero-positivity to T. gondii, Rubella, CMV and HSV-2 was 26.6/9.2 \%, 9.2/78.9\%, $17.4 / 58.7 \%$, and $4.6 / 35.8 \%$ respectively. There was an increased percentage of CMV infection. There was increased number of recent Toxoplasma infection in comparison to other studies conducted.

Conclusion: A previous history of pregnancy wastage and the serological reaction for TORCH infections during current pregnancy must be considered while managing $\mathrm{BOH}$ cases so as to reduce the adverse fetal outcome.
\end{abstract}

\section{INTRODUCTION}

Primary infection caused by TORCH- Toxoplasma gondii, Rubella virus, cytomegalovirus (CMV), and Herpes simplex virus (HSV) - is the major cause of infection with peri- and post natal morbidity. The effect of the infection not only leads to single or repeated fetal loss but also recentand delayed complications to the mother and the fetus who survives.

The incidence of infection by toxoplasma, rubella and

\section{Correspondence:}

Dr. S.V.Pradhan, MBBS, MD

Advance Pathology Laboratory Pvt. Ltd.

Birtamode, Jhapa, Nepal

Email:seemavpradhan@gmail.com
CMV, and Herpes varies in different parts of the world. These maternal infections are initially unapparent or asymptomatic and are, thus, difficult to diagnose on clinical grounds. Therefore, diagnosis of acute TORCH infection in pregnant women is usually established by demonstration of seroconversion in paired sera or by demonstration of specific IgM antibodies. ${ }^{1}$ This study is conducted to analyze the epidemiological and serological aspect of TORCH infection in eastern Nepal.

\section{MATERIAL AND METHODS}

The population under study represents from major part of Eastern Nepal- hilly and terai regions of Jhapa , Illam, Panchthar, Taplejung and parts of Morang the 
study was conducted in Advance Pathology Laboratory of Birtamode during the period of 1.5 years from 2012 - 2014. A total of 109 cases with history of unfavorable pregnancy outcome were included in the study. All cases coming to the laboratory with history of fetal loss were included in this study. The patients with other known factors of fetal loss were not included in the study.

\section{RESULTS}

The study included 109 patient from different parts of eastern Nepal including Panchthar, Ilam, Jhapa, and part of Morang and Taplejung.The youngest person included in the study was of $20 \mathrm{yrs}$ of age andoldest being 40 years. Majority $66 \%$ of the patients were between the age group of 21-30 yrs (fig.1).

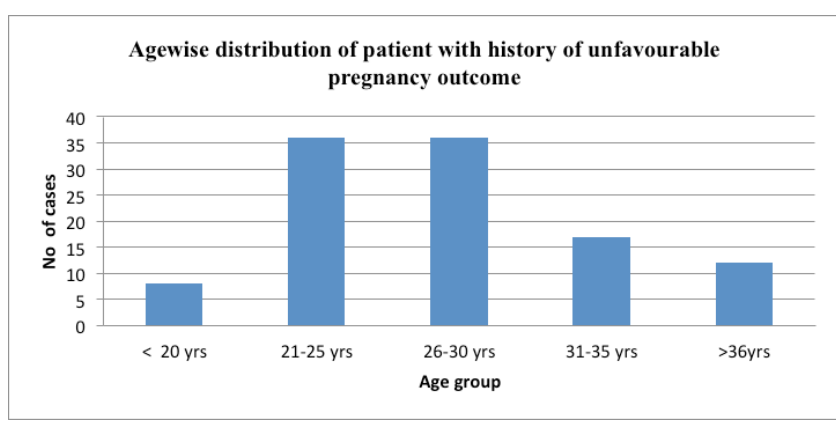

Figure 1. Age wise distribution of patients

The predominant populations of the study group fall in the Brahmin and Chettri population (67\%) with 14\% Mongolians. The Mongolians included Rai, Limbu, Tamang, Magar.

Out of total 109 cases $28(25.6 \%)$ shows recent infection by Toxoplasma Gondi, $4.5 \%$ by Rubella, $2.7 \%$ of CMV and $0.9 \%$ of HSV. The study showed positive reactivity to more than one infection. Our study shows an increased prevalence of cytomegalovirus infection with IgG positivity in $72.5 \%$ of cases (Table 1 ).

Reinfection by rubella was seen in $12.8 \%$ of cases. Two Cases of rubella with equivocal $\operatorname{IgG}$ were repeated after 14 days and shows positivity. Other four equivocal cases were lost to follow up.

Patients were also categorized into subgroups according to their past obstetric history. The categories include patient with Bad obstetric history (BOH- cases with $>2$ abortion),

\section{Table 1: Serology findings :IgG and IgM Positivity}

\begin{tabular}{lcccc} 
& IgG & IgM & IgG+IgM & Equivocal \\
\hline Toxoplasma & 9 & 28 & 1 & 2 IgM \\
Rubella & 50 & 5 & 14 & 6 IgG \\
CMV & 79 & 3 & 7 & 1 IgM \\
Herpes & 38 & 4 & 1 & \\
& & & &
\end{tabular}

spontaneous abortion, history of intrauterine fetal death, andpresence of congenital anomaly. $77.9 \%$ cases had history of single abortion, $36.7 \%$ had more than 2 abortions. 5 cases i.e $4.6 \%$ had history of IUFD. Congenital anomaly with fetal loss was seen in one young female of 20 years. The figure 2 shows the comparison of positive IgG serology of Toxoplasma, Rubella, CMV and HSV with maternal obstetric history. The finding showed an increase of CMV in all cases.

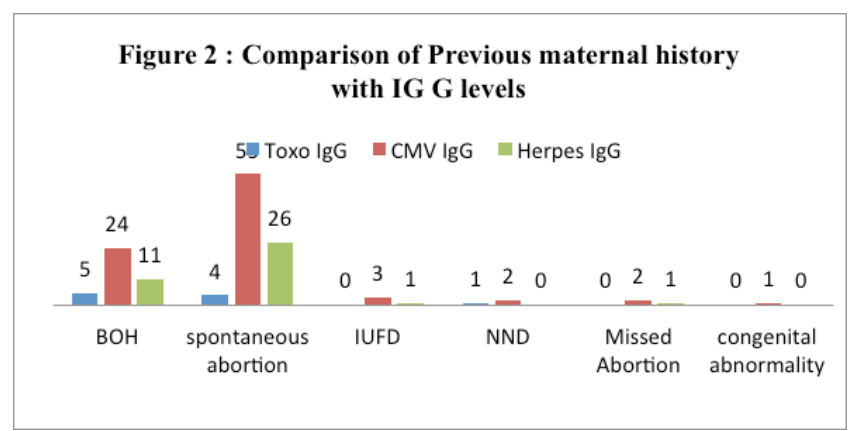

Figure 2 : Comparison of Previous maternal history with $I G$ levels

\section{DISCUSSION}

Infections by Toxoplasma gondii, Rubella, CMV and Herpes infection are known factors for perinatal morbidity and mortality.

\section{Toxoplasmosis}

Toxoplasmosis is an infection by obligate intracellular protozoan parasite Toxoplasma gondii. It is one of the most prevalent chronic infections affecting one third of world's population, ${ }^{2-4}$ characterized by nonspecific symptoms like fever, malaise and lymphadenopathy. Maternal screening is recommended when fetal abnormalities like hydrocephaly, anatomic abnormalities of the central nervous system, symmetric fetal growth restriction, and nonimmune hydrops are observed.

Nowadays, a major effort has been made toward diagnosing recently acquired toxoplasma infection in the pregnant woman and congenital infection in the fetus and newborn. The detection of anti-toxoplasma antibodies by ELISA (enzyme-linked immunosorbent assay) methods is commonly performed.

The presence of elevated levels of Toxoplasma specific IgG antibodies indicates infection has occurred at some point. Detection of Toxoplasma-specific IgM antibodies has been used as an aid in determining the time of infection, but IgM antibodies have been reported to persist for up to 18 months post infection. Negative IgM results are reassuring, whereas positive results should be interpreted carefully, and followed by serial titers at least 3 weeks apart. ${ }^{5}$ Acute toxoplasmosis 
is diagnosed rarely by detecting the parasite in body fluids, tissue, or secretions. ${ }^{6}$

There are different Toxoplasma seropositivity reports from all over the world. The population of Turkish childbearing age women has the seropositivity of $\mathrm{T}$. gondii as $1.34 \%$ for IgM and $24.6 \%$ for IgG. In Qatar among 823 women of childbearing age the $\mathrm{T}$. gondii $\operatorname{IgG}$ and $\operatorname{IgM}$ was $35.1 \%$ and $5.2 \%$ respectively In another study in Iran, 247 of the 553 pregnant women were found to be positive for $\operatorname{IgG} .^{7}$ In a study conducted in India, anti-Toxoplasma IgG antibodies were detected in $15.33 \%$ cases, while $3 \%$ had positive anti-Toxoplasma IgM..$^{8-10}$ The prevalence of toxoplasmosis in $\mathrm{BOH}$ is known to be significantly higher than those without it. A recent study from Chandigarh reports rising seropositivity to toxoplasma in women with $\mathrm{BOH} .^{7}$ In our study we found an increase in the recent infection with $28.6 \%$ of toxoplasma cases in comparison to the other studies conducted in Nepal.

\section{Rubella}

The estimated risks of fetal damage following primary infection is highest when infection occurs in the first 8 weeks after the last menstrual period, when $90-100 \%$ of fetuses will become infected and up to $100 \%$ of the infected fetuses will develop major clinical defects. Such defects typically include those affecting the heart, vision and auditory function. The risk of both fetal infection and the incidence and severity of congenital defects progressively declines after the first trimester and the risk of any defects after 17 weeks gestation is rare.

The risk of fetal infection following maternal reinfection has been variably estimated as 0 to 30 percent. Most reinfections are asymptomatic. When a pregnant patient has contact with known or suspected rubella case, or has a nonspecific viral-like illness with or without rash, clinicians are advised to perform serial rubella antibody tests, regardless of vaccination status.

The distinction between primary and secondary infection is ultimately in the hands of the serology laboratory. Sero-epidemiological studies have shown that 10-20\% of women in childbearing age in India are susceptible to rubella infection. Infection with Rubella during pregnancy may lead to congenital malformation in $10-54 \%$ of cases. ${ }^{6}$

A single IgG antibody measurement of less than $10 \mathrm{IU} / \mathrm{mL}$ would be reported as showing no evidence of prior rubella vaccination or infection. A significant rise in $\mathrm{IgG}$ level is required to diagnose rubella reinfection serologically. Unlike primary infection, reinfection is characterized by high avidity antibody binding. In our study, the IgG titre of Rubella shows a wide variation in the serum levels with maximum patient having the $\mathrm{IgG}$ between $10-200 \mathrm{IU} / \mathrm{ml}$. as seen in the following figure 3 . The highest value was 1400

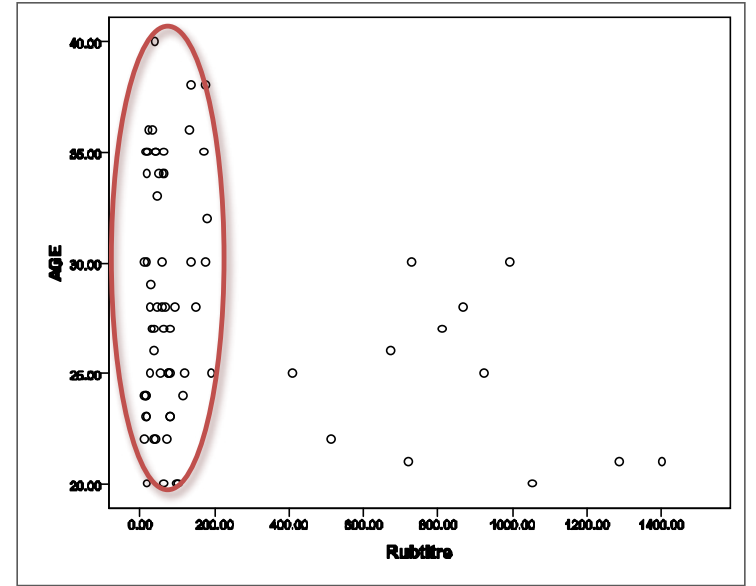

Figure 3: Distribution of serum IgG values Rubella

$\mathrm{IU} / \mathrm{ml}$ noted in a case of reinfection.

Cytomegalovirus

Cytomegalovirus (CMV) is a ubiquitous DNA herpesvirus that causes a wide variety of clinical manifestations. It is the most common congenital viral infection, with birth prevalence of about $0.5 \%$ (range 0.2 to $3 \%$ ). Maternal seroprevalences of CMV antibodies derived from different studies, varied from $0.15 \%$ to $0.5 \%$ in Europe, whereas those in North America varied from $0.42 \%$ to $1.4 \%$. In Africa and Asia, the reported incidences vary from $0.5 \%$ in Japan, $1.38 \%$ in Ivory Coast, and $1.8 \%$ in Taiwan, in the presence of a very high rate of preexisting maternal immunity $(90 \%-100 \%) .{ }^{11,12}$

In the studied conducted in Nepal an infection rate of 34\% was found. In Our study also shows Majority of the cases $72.4 \%$ cases with positivity for CMV IgG, with $4.5 \%$ of recent infection. ${ }^{3,4}$

The factors responsible for transmission to the fetus and severity of congenital CMV infection are not well understood. Preexisting maternal CMV seropositivity substantially decreases, but does not completely eliminate, fetal infection, suggesting partial protection from maternal immunity.

Although the majority of congenital infections are asymptomatic, 5 to $20 \%$ of infants born to mothers with primary CMV infection are overtly symptomatic. These children have a mortality rate of about $5 \%$, and severe neurologic morbidity occurs in 50 to $60 \%$ of survivors. ${ }^{11,12}$ Asymptomatic infants are also at risk of developing longterm neurodevelopment morbidity, but the risk is much lower than in symptomatic neonates.

\section{Herpes simplex virus}

Herpes simplex virus (HSV) is a ubiquitous, enveloped, 
and double stranded DNA virus, belonging to the family of Herpesviridae transmitted across mucosal membranes and nonintact skin that migrate to nerve tissues, where they persist in a latent state. HSV-1 predominates in orofacial lesions, and it is typically found in the trigeminal ganglia, whereas HSV-2 is most commonly found in the lumbosacral ganglia. ${ }^{13,14}$

There is an increased incidence of genital herpes in pregnancy. The seroprevalence varies from $7.6 \%$ to $22 \%$ seroprevalence. ${ }^{15}$ Nevertheless it is lower than that reported among pregnant women in other countries. The acquisition of genital herpes during pregnancy has been associated with spontaneous abortion, intrauterine growth retardation, preterm labour, and congenital and neonatal herpes infections. ${ }^{16}$

Intrauterine viral transmission is highest during the first 20 weeks of gestation leading to abortion, stillbirth, and congenital anomalies. The perinatal mortality is $50 \%$. The risk of neonatal infection varies from $30 \%$ to $50 \%$ for $\mathrm{HSV}$ infections that onset in late pregnancy (last trimester), whereas early pregnancy infection carries a risk of about $1 \% .{ }^{17}$ In our study Herpes simplex IgG was seen in $0.9 \%$ of the cases with only one case of recent infection.

\section{CONCLUSION}

Maternal infections are a well-known cause of fetal morbidity and fetal loss. The prevalence of TORCH infection in our part of the country is relevant. The loss can be avoided by early identification and efficient treatment.

\section{ACKNOWLEDGEMENT}

I hereby thank all the Gynaecologists specially Dr. SP Huzdar, Dr. B Budhathoki, Dr. M Tiwari for their support and my team for their contribution.

\section{REFERENCES}

1. Padmavathy M, Mangala G, Malini Jet al; Seroprevalence of TORCH Infections and Adverse Reproductive Outcome in Current Pregnancy with Bad Obstetric History J Clin Biomed Sci 2013;3:62-71.

2. Jones JL, Kruszon-Moran D, Wilson M, McQuillan G, Navin T, McAuley JB. Toxoplasma gondii infection in the United States: seroprevalence and risk factors. Am J Epidemiol 2001;154:357-65.

3. Kumari N, Morris N, Dutta R. Is screening of TORCH worthwhile in women with bad obstetric history? An observation from eastern Nepal. Journal of Health, Population, and Nutrition 2011;29:77-80. Crossref

4. Acharya D, Shrestha A, Bogati B, Khanal K, Shrestha S, Gyawali P. Serological Screening of TORCH Agents as an Etiology of Spontaneous Abortion in Dhulikhel Hospital, Nepal. American Journal of Biomedical and Life Sciences 2014;2:34-9. Crossref
5. Wilson M, McAuley JM. Toxoplasma. In: Murray PR, Baron ES, Pfaller MA et al., eds. Manual of Clinical Microbiology, 7th Ed.Washington, DC: ASM Press, 1999. pp 1374-82.

6. Montoya JG. Laboratory diagnosis of Toxoplasma gondii infection and toxoplasmosis. J Infect Dis 2002;185:S73-82.

7. Tekkesin N. Diagnosis of toxoplasmosis in pregnancy: a review. HOAJ Biology 2012;1:1-9. Crossref

8. Aggarwal A, Diddi K, Malla N,et al. Serological screening for antenatal toxoplasma infection in India. Indian J Med Microbiol 2010;28:143-6.

9. Surpam RB, Kamlakar UP, Khadse RK, et al. Serological study for TORCH infections in women with bad obstetric history. J Obstet Gynecol India 2006;56:41-3.

10. Kaur RN, Gupta D, Nair M. Kakkar, Mathur MD. Screening for TORCH infections in pregnant women: A report from Delhi. Southeast Asian J. Trop. Med. Public Health 1999;30(2):284-86.

11. Kenneson A, Cannon MJ. Review and meta-analysis of the epidemiology of congenital cytomegalovirus infection. Rev Med Virol 2007;17:253. Crossref

12. Istas AS, Demmler GJ, Dobbins JG, Stewart JA. Surveillance for congenital cytomegalovirus disease: a report from the National Congenital Cytomegalovirus Disease Registry. Clin Infect Dis 1995;20:665. Crossref

13. Gupta R, Warren T, and Wald A. "Genital herpes," The Lancet 2007;370:2127-37. Crossref

14. Gianluca Straface, Alessia Selmin, Vincenzo Zanardo, Marco De Santis, Alfredo Ercoli, and Giovanni Scambia. "Herpes Simplex Virus Infection in Pregnancy," Infectious Diseases in Obstetrics and Gynecology vol. 2012, Article ID 385697, 6 pages, 2012. Crossref

15. Suligoi B, Cusan M, Santopadre P, et al., "HSV-2 specific seroprevalence among various populations in Rome, Italy. The Italian herpes management forum," Sexually Transmitted Infections 2000;76:213-4. Crossref

16. M. Arvaja, M. Lehtinen, P. Koskela, M. Lappalainen, J. Paavonen, and T. Vesikari, "Serological evaluation of herpes simplex virus type 1 and type 2 infections in pregnancy," Sexually Transmitted Infections 1999;75:168-71. Crossref

17. D. Money and M. Steben, "Guidelines for the management ofherpes simplex virus in pregnancy," International Journal of Gynecology \& Obstetrics 2009;104:167-71. Crossref 